Courrier du Centre International Blaise-

Pascal

38-39 | 2017-2018

Varia

\title{
Bibliographie pascalienne 2017-2018
}

\section{(2) OpenEdition}

1 Journals

Édition électronique

URL : https://journals.openedition.org/ccibp/1528

DOI : 10.4000/ccibp.1528

ISSN : 2493-7460

Éditeur

Centre international Blaise Pascal

\section{Édition imprimée}

Date de publication : 1 janvier 2019

Pagination : 199-202

ISBN : 978-2-84516-897-8

ISSN : 0249-6674

\section{Référence électronique}

«Bibliographie pascalienne 2017-2018», Courrier du Centre International Blaise-Pascal [En ligne], 38-39 |

2017-2018, mis en ligne le 21 juin 2021, consulté le 03 mai 2022. URL : http://

journals.openedition.org/ccibp/1528; DOI : https://doi.org/10.4000/ccibp.1528

Ce document a été généré automatiquement le 3 mai 2022.

Centre international Blaise Pascal 


\section{Bibliographie pascalienne 2017-2018}

1 Cette bibliographie tient compte de toutes les publications qui ont été signalées au Centre International Blaise-Pascal.

\section{BIBLIOGRAPHIE}

\section{Année 2017}

ALBANESE Jr Ralph, « La réception critique des Pensées de Pascal à l'école républicaine », Papers on French Seventeenth Century Literature, 48, 2017, p. 113-126.

BAHIER-PORTE Christelle, MOREAU Pierre-François, REGUIG Delphine, Liberté de conscience et arts de penser (XVI ${ }^{e}$-XVIII ${ }^{e}$ siècle). Mélanges en l'honneur d'Antony McKenna, Paris, Champion, 2017.

BAS-OSTROWIECKI, « Continuité de l'inconstance et irruption de la perpétuité : paradoxes de l'histoire chez Pascal », dans BAHIER-PORTE Christelle, MOREAU Pierre-François, REGUIG Delphine (dir.), Liberté de conscience et arts de penser (XVI -XVIII siècle). Mélanges en l'honneur d'Antony McKenna, Paris, Champion, 2017, p. 301-311.

BELLANTONE Andrea, «La liberté au principe », Chestov-Pascal, Cahiers Léon Chestov, 17-2017, Artix Print House, 2017, p. 111-122.

BISCHOFF Jean-Louis, Pascal et la pop-culture, Paris, L'Harmattan, 2017.

BORGHERO Carlo, « « L'Art de penser, le pyrrhonisme, la connaissance des faits », dans BAHIERPORTE Christelle, MOREAU Pierre-François, REGUIG Delphine (dir.), Liberté de conscience et arts de penser (XVI ${ }^{e}-\mathrm{XVIII}{ }^{e}$ siècle). Mélanges en l'honneur d'Antony McKenna, Paris, Champion, 2017, p. 313-326. 
BROADIE Alexander, «The Role of Reason in the Assent of Faith. Pascal, Shestov and the Late Medieval Background », Chestov-Pascal, Cahiers Léon Chestov, 17-2017, Artix Print House, 2017, p. 57-71.

CAWS Mary Ann, Blaise Pascal. Miracles and reason, London, Reaktion books, 2017.

Courrier du Centre International Blaise Pascal, 38, 2016.

Chestov-Pascal, Cahiers Léon Chestov, 17-2017, Artix Print House, 2017.

DELAUNAY Guy, Blaise Pascal en quête d'une apologétique renouvelée, Louvain-la-Neuve, EME Éditions, 2017.

DESCOTES Dominique, «Chronique », Courrier du Centre International Blaise Pascal, 38, 2016, p. 3-20. DESCOTES Dominique, « L'égalité chez Pascal », dans BAHIER-PORTE Christelle, MOREAU PierreFrançois, REGUIG Delphine (dir.), Liberté de conscience et arts de penser (XVI ${ }^{e}$-XVIII ${ }^{e}$ siècle). Mélanges en l'honneur d'Antony McKenna, Paris, Champion, 2017, p. 265-299.

FERREYROLLES Gérard, «Un pascalien au pays de Chestov », Chestov-Pascal, Cahiers Léon Chestov, 17-2017, Artix Print House, 2017, p. 3-13.

FOTIADE Ramona, « Foi et raison chez Léon Chestov et Blaise Pascal », Chestov-Pascal, Cahiers Léon Chestov, 17-2017, Artix Print House, 2017, p. 15-29.

GALLARD Pierre-Yves, «Implicite et stratégies argumentatives : les paradoxes des Provinciales », dans ANQUETIL Sophie, ELIE-DESCHAMPS Juliette et LEFEBVRE-SCHOELLER Cindy, Autour des formes implicites, Rennes, Presses Universitaires de Rennes, 2017, p. 127-135.

GRASSET Bernard, Pascal, Paris, Ellipses, coll. « Connaître en citations », 2017.

GRASSET Bernard, «Léon Chestov et Blaise Pascal, lecteurs de l'Écriture. D'une foi sans raisons aux raisons de la foi », Chestov-Pascal, Cahiers Léon Chestov, 17-2017, Artix Print House, 2017, p. 31-55.

JERPHAGNON Lucien, L'au-delà de tout, Paris, Robert Laffont, coll. « Bouquins », 2017.

GROS Jean-Michel, «L'athéisme à la croisée des chemins. Dévots et libertins : Pascal et Cyrano, Leibniz et Bayle », Libertinage, athéisme et incrédulité, 2, Littératures classiques, nº 93, 2017, p. 9-32.

JULLIARD Jacques, L'esprit du peuple, Paris, Robert Laffont, 2017.

LUEZ Philippe, Port-Royal et le jansénisme. Des religieuses face à l'absolutisme, Paris, Belin, 2017.

McKENNA Antony, Pascal et son libertin, Paris, Garnier, 2017.

MERKER Claude, « La Lettre à Huygens. Explication de texte », Courrier du Centre International Blaise Pascal, 38, 2016, p. 21-38.

PARISH Richard, « État présent. Blaise Pascal », French studies, 71, 4, 2017, p. 539-550.

PARLEBAS Jean-Claude, « Un admirateur de Blaise Pascal : le professeur Reijer Hooykaas », Courrier du Centre International Blaise Pascal, 38, 2016, p. 39-44.

REGUIG Delphine, «L'auctorialité dans Les Provinciales », dans MARTIN Christophe (dir.), Raconter d'autres partages : littérature, anthropologie et histoire culturelle: Mélanges offerts à Nicole JacquesLefevre, Lyon, ENS Éditions, 2017, p. 147-161.

SCHNEIDER Line, « Pascal », Pascal dans le siècle, 158, 2017, p. 409-411. 
SORYANO Line, «Pensées elliptiques de Pascal », dans ANQUETIL Sophie, ELIE-DESCHAMPS Juliette et LEFEBVRE-SCHOELLER Cind (dir.)., Autour des formes implicites, Rennes, Presses Universitaires de Rennes, 2017, p. 137-148.

WIEL Véronique, Usage du monde et liberté à l'âge classique, Paris, Champion, 2017.

\section{Année 2018}

ANDRÉ Gérard, « Artus Gouffier de Roannez, entrepreneur de la "nouvelle navigation de la Seine" entre Troyes et Nogent », La Vie en Champagne, 93, janvier-mars 2018, p. 25-37.

BARTA Paul, Pascal's wager, Cambridge, Cambridge University Press, 2018.

BOUCHARD Hélène, Pascal et la mystique, Paris, L'Harmattan, 2018.

CARMONA Michel, Port-Royal, Paris, Fayard, 2018.

DESCOTES Dominique, « Alexandre Koyré and Blaise Pascal », dans PISANO Raffaele, AGASSI Joseph et DROZDOVA Daria, Hypotheses and Perspectives in the History and Philosophy of Science. Homage to Alexandre Koyré 1892-1964, Springer, 2018, p. 105-122.

GRASSET Bernard, « Chemins de philosophie biblique », Revue des Sciences Religieuses, 92/1, 2018, p. 99-119.

HORINO Masamitsu, «Introduction aux études génétiques sur les Copies des Pensées de Pascal », Acta humanistica et scientifica Universitatis Sangio Kyotiensis, Humanities series, $\mathrm{n}^{\circ}$ 51, Kyoto Dangyo University, mars 2018, p. 349-363.

NORO Yasushi, Une vie à la trace. Amable Bourzeis, écrivain (1606-1672), Paris, Garnier, 2018.

PAILLAT Sylvie, « Entretien avec Bernard Grasset », La revue littéraire, 71, janvier-février 2018, p. 115-129.

ROMEO Maria-Vita, « Un foyer de recherches sur Pascal », XVII siècle, $\mathrm{n}^{\circ}$ 281, octobre 2018, p. 663-673.

THIROUIN Laurent, « La république et le nombre : Pascal penseur de la pluralité », Chroniques de Port-Royal, Port-Royal et la république : 1940-1629 ?, Paris, 2018, p. 217-236.

\section{Année 2019}

COUMET Ernest, Euvres d'Ernest Coumet, Tome 2, éd. Catherine Goldstein, Besançon, Presses Universitaires de Franche-Comté, 2019.

FRIGO Alberto, « Pascal, admirateur frustré », Michel Eyquem de Montaigne, Le Point, Hors-série, 25, juin-juillet 2019, p. 78-79.

FUMAROLI Marc, Partis pris. Littérature, esthétique, politique, Paris, Robert Laffont, coll. «Bouquins ", 2019.

FUMAROLI Marc, « Pascal », Partis pris. Littérature, esthétique, politique, Paris, Robert Laffont, coll. «Bouquins », 2019, p. 181-184. 
INDEX

Mots-clés : bibliographie, Pascal

Keywords : bibliography, Pascal 